\title{
Adaptable Energy Systems Integration by Modular, Standardized and Scalable System Architectures: Necessities and Prospects of Any Time Transition
}

\author{
Jonas Hinker ${ }^{1} *$ (D), Thomas Wohlfahrt ${ }^{1}$, Emily Drewing ${ }^{2}$, Sergio Felipe Contreras Paredes ${ }^{1}$, \\ Daniel Mayorga González ${ }^{1}$ and Johanna M. A. Myrzik ${ }^{1}$ \\ 1 Institute of Energy Systems, Energy Efficiency and Energy Economics $\left(\mathrm{ie}^{3}\right)$, TU Dortmund, \\ Emil-Figge-Str. 70, 44227 Dortmund, Germany; thomas.wohlfahrt@tu-dortmund.de (T.W.); \\ sergio.contreras@tu-dortmund.de (S.F.C.P.); daniel.mayorga@tu-dortmund.de (D.M.G.); \\ johanna.myrzik@tu-dortmund.de (J.M.A.M.) \\ 2 Chair of Sociology, Labour and Economics, Ruhr-Universität Bochum, Universitätsstr. 150, 44801 Bochum, \\ Germany; emily.drewing@rub.de \\ * Correspondence: jonas.hinker@tu-dortmund.de; Tel.: +49-231-755-3022
}

Received: 22 February 2018; Accepted: 2 March 2018; Published: 7 March 2018

\begin{abstract}
Energy conversion and distribution of heat and electricity is characterized by long planning horizons, investment periods and depreciation times, and it is thus difficult to plan and tell the technology that optimally fits for decades. Uncertainties include future energy prices, applicable subsidies, regulation, and even the evolution of market designs. To achieve higher adaptability to arbitrary transition paths, a technical concept based on integrated energy systems is envisioned and described. The problem of intermediate steps of evolution is tackled by introducing a novel paradigm in urban infrastructure design. It builds on standardization, modularization and economies of scale for underlying conversion units. Building on conceptual arguments for such a platform, it is then argued how actors like (among others) municipalities and district heating system operators can use this as a practical starting point for a manageable and smooth transition towards more environmental friendly supply technologies, and to commit to their own pace of transition (bearable investment/risk). Merits are not only supported by technical arguments but also by strategical and societal prospects like technology neutrality and availability of real options.
\end{abstract}

Keywords: energy infrastructure design; system architecture; energy transition; district heating systems (DHS); energy hubs; distributed multigeneration (DMG); multi-energy systems (MES); urban energy systems (UES); community energy; societal prospects

\section{Introduction}

Many countries show a high heat demand [1] which goes along with significant carbon emissions. One means to decarbonize the heat supply is given by more efficient combined heat and power (CHP) approaches. While CHP is one specific technology that integrates heat and power delivery, other grids can be considered for integration as well: For instance, natural gas can be stored in pipelines with neglectable losses $[2,3]$ while electricity can be transmitted efficiently over long distances. Besides, district heating systems can be used as short- and medium term storage for a more efficient dispatch of conversion units [4]. In summary, lower costs and carbon emissions generation may be achieved by integrating different energy systems, and the term energy systems integration has therefore received a lot of attention recently [5].

However, despite thinkable benefits, planning of integrated energy systems generally involves a high level of uncertainty concerning future demand, prices and regulation. Uncertainty thus translates 
into financial risk and threatens the profitability. Naturally, risk-averse investors do favour simpler projects with a guaranteed profitability. Therefore, only few integrated systems have actually been implemented in the real world. Looking into the profitability of planned projects, the analyses often build on tight calculations [6,7]. For instance, the return on invest is often low while payback times are found to be high, even if additional business cases like the provision of operating reserve to connected grids from such systems is considered. However, another reason for low adoption rates is the multitude of risks and uncertainties that threaten these business cases.

\subsection{Literature Review of the Investment Planning Problem}

In general, financial and non-financial risks have been systematically considered in portfolio planning and optimal dispatch by a wide range of optimization techniques. and numerous approaches to deal with uncertainty can thus be found in the literature (cf. $[8,9]$ for more extensive reviews): One way to deal with uncertainty is the application of mean-variance portfolio theory and a thorough consideration of risk scenarios [10-12]. Other approaches use mixed integer linear programming to optimize a portfolio of conversion units and their sizing and siting. Simpler approaches study the optimal investment for one investment period, while more sophisticated approaches additionally tell the optimal time of invest, which is thus called optimal multi-stage investment [13-18]. If multiple objectives cannot be weighted beforehand but must still be optimized simultaneously, evolutionary optimization can be used to find the multi-dimensional pareto frontiers. This has been successfully applied to the investment planning problem in, e.g., [19]. A special case is given for so called cooperative or coalitional planning, where the optimal solution is negotiated by different distributed subsystems with differing potentials for, e.g., the installation of renewable generation, and the optimal siting is an immanent feature of this problem formulation [20,21]. Instead of estimating underlying risk variables for certain future scenarios, robust optimization can be used to determine the optimal portfolio over a range of thinkable values [22]. In addition, if underlying probability density functions of stochastic parameters are known, stochastic programming techniques help to better incorporate uncertainties when optimizing the generation portfolio [23].

In an ideal world, uncertainty would in fact be managed by implementing new technologies in small portions, i.e., incrementally. As time progresses, energy demand, prices and regulation do not only evolve but do also become more assured, i.e., the variance of prices and demand is reduced. This way, the (remaining) riskiness of the (remaining) project is reduced step by step by growing know-how and expertise from the parts already implemented, and by diminishing uncertainty of the business case. The course of implementation can thus be changed by the management, so that the final realization might differ from the initially projected system. This way of managing a business by gradually learning from actual realizations of uncertain parameters is called real options management, and a successor of multi-stage investment. Real options-based approaches for optimal multi-stage investment planning into integrated energy systems have been proposed and applied in case studies [24-26].

In summary, optimization approaches often explicitly consider the optimal timing of investments by different means, but most approaches presented above tell the optimum from today's perspective, and means to deliberately readjust decisions multiple times are not widespread. In this context, it is also worth noting that the term bridging systems has been presented in the context of planning of integrated energy systems in [27], and the corresponding Figure 1 indicates that the optimal path of realization stages towards a final optimum might change in the course of time, which clearly underlines the necessity to deal with the problem of any time transitions. 


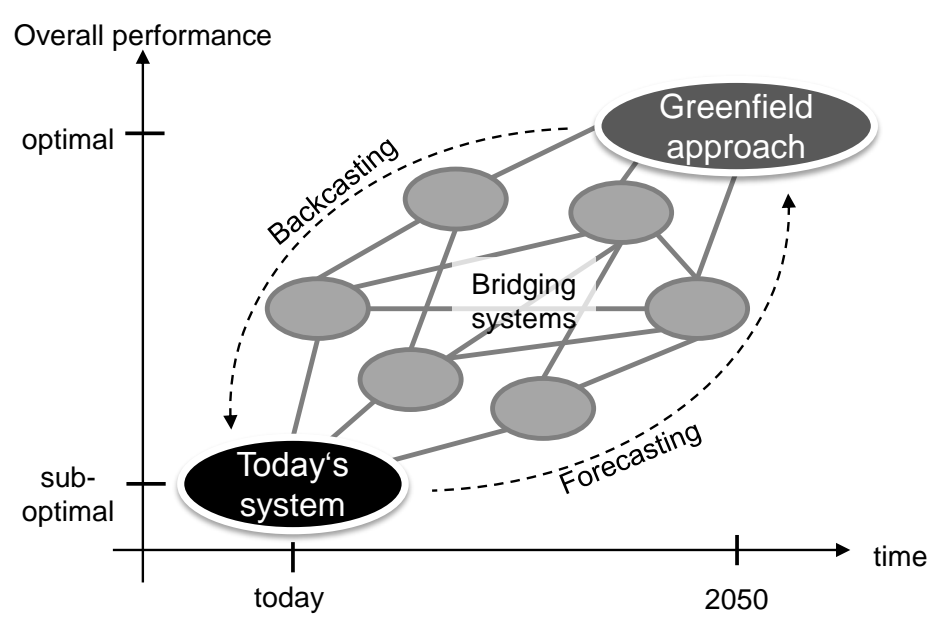

Figure 1. Vision of transition paths to final greenfield optimality, adapted from [27].

\subsection{Research Gap and Contribution of This Work}

Many researchers have analyzed intermediate steps of the investment planning problem to improve supply systems step by step. Furthermore, the need to readjust the optimal path from time to time (i.e., by bridging systems) has been clearly stated. However, in reality, such visions [27] and case studies $[10,26]$ remain rather theoretic for the following reasons: Investments in generation capacity are characterized as large-scale infrastructure projects, which are defined by high capital intensity and long investment periods (e.g., depreciation times of 50 years for large power stations). Most strikingly, such an investment is normally neither scalable nor separable. While the lack of scalability means incremental investments are not possible, inseparability also means the entire investment has to be done by one investor. Therefore, the current situation in investment planning for energy supply is best described as all-or-nothing and atomic.

To this end, this paper will contribute with a suggestion of tangible guiding principles that will render a step-wise and thus more feasible transition possible. The conceptual architecture is best thought of as an infrastructure platform. Once this platform has been implemented, different supply technologies can be integrated successively. Therefore, the proposed architecture involves a paradigm change for future planning and optimization of energy supply.

In this work, we mainly address (among others) municipal heat suppliers, district heating system operators and policy makers to propose a novel technical system architecture. This architecture is meant to function as a cost-effective enabler of arbitrary transition paths for the physical implementation of energy supply. It therefore decreases the barriers to optimally readjust a given energy supply system in the course of time. Consequently, suggested visions and investment planning approaches $[10,26,27]$ become feasible again because changes to the portfolio are rendered possible at any time (hence the title), which is a novelty.

To summarize it, the main contributions of this paper are thus:

- A technical concept building on modularity, standardization and scalability is presented at a manageable level of abstraction but including detailed notes on implementation (Section 2).

- The notion of adaptability is introduced in the context of sustainable energy infrastructure (Section 2).

- A case study shows an exemplary system's evolution, which is enabled by the presented architecture (Section 3).

- Positive technical, strategical and societal prospects are discussed (Section 4).

- The connection to other concepts and visions of energy systems integration is shown to highlight compatibility and thus direct implementability (Appendix A). 


\section{Envisioned Technical System Architecture of Future Infrastructure and Supply}

This section presents the developed concept of future energy systems integration. Although generally adaptable to other energy carriers, the presentation in this paper is focused on the provision of heat and electricity in proximity to the customer.

\subsection{Overview of Basic Elements of the Architecture}

As shown in Figure 2 the system comprises different classes of conversion and storage units. Conversion units are classified by the type of conversion, which is their input-output-connection of different energy carriers. For instance, combined heat and power (CHP) is one class of conversion units as these units convert natural gas into heat and electricity. An electric heat pump, however, can be characterized as belonging to another such class, because electricity is converted to heat. Other classes might not include a direct conversion, but store energy by charging and discharging. Such elements are included in the classification.

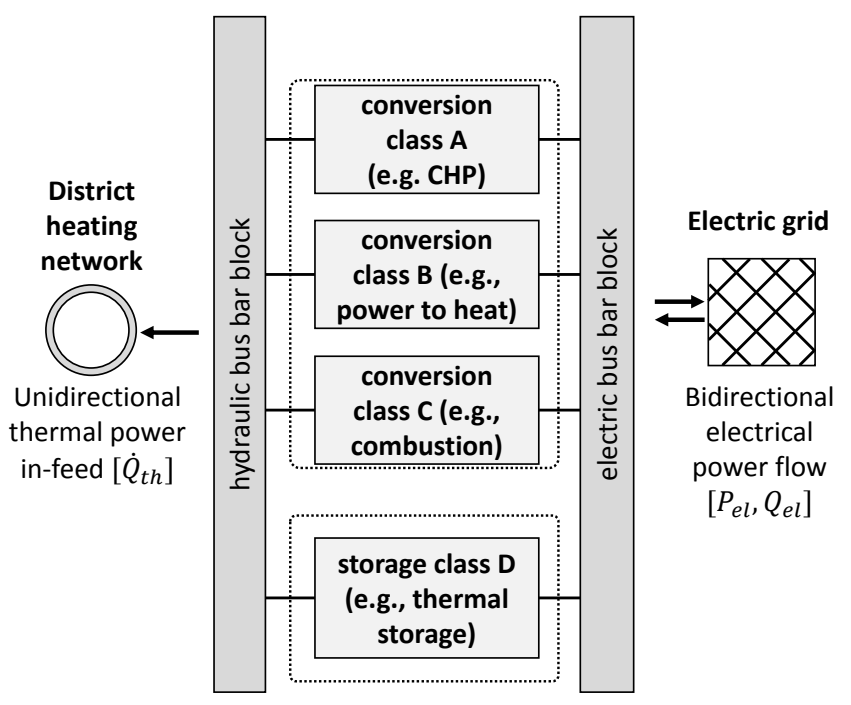

Figure 2. Abstract perspective on key elements of the architecture: energy conversion and storage units are modularized and can easily be snapped into the platform to connect to different networks.

In reality, the realization of conversion classes is achieved by installation of physical conversion units. Of course, a mix of different conversion units from different vendors might still belong to the same class of conversion units. Naturally, this architecture does not require the installation of certain classes or even conversion units. Therefore, the mix of conversion units can be chosen by the corresponding system integrator. Note that a mix of conversion and storage units is also referred to as a unit portfolio (or even portfolio in short).

As discussed in the introduction, even if a certain unit portfolio is envisioned for the future (Figure 1), it is possible that significant changes have to be made to the installation. To account for this inherent management flexibility, the architecture also requires that a significant share of the space within the physical platform is devoted to future extensions or changes.

All units are therefore connected to the distribution infrastructure. The chosen portfolio is able to cover a given electrical and thermal supply task. On the left hand side of the conversion units (both in Figures 2 and 3), there is a bus bar-like hydraulic configuration box that is itself directly connected to the district heating network (DHS). 


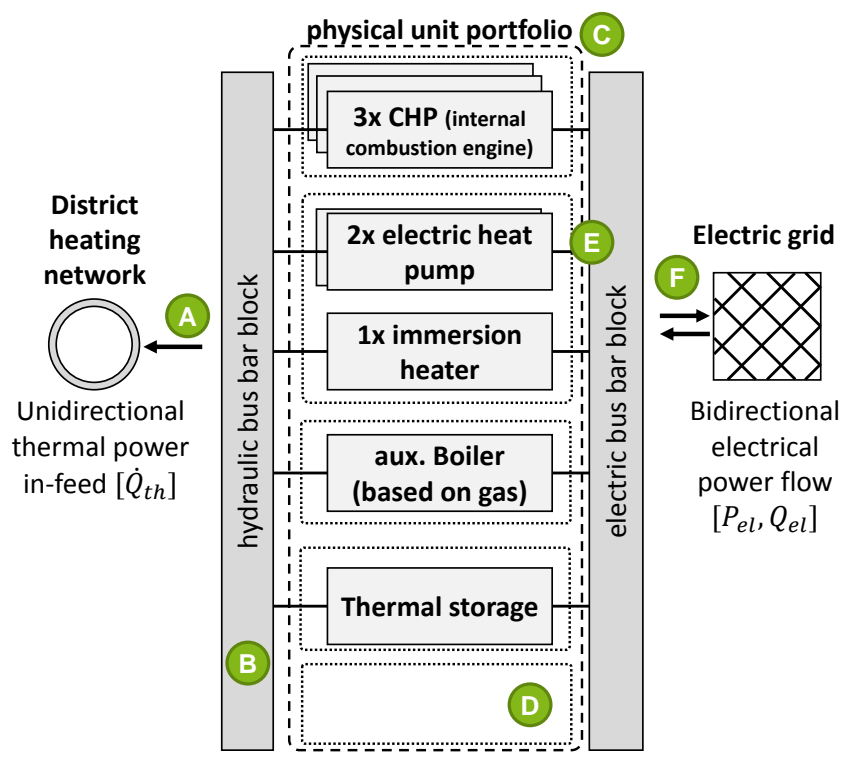

(a)

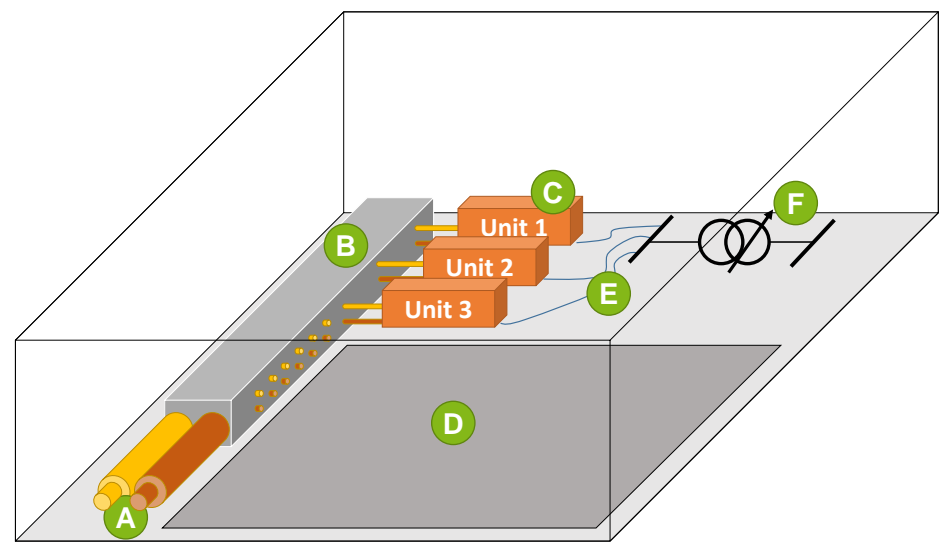

A Connection to district heating system

B Flexible hydraulic patch panel

C) Installed units (20"/40" containers)

D Space for further extensions

E Electric connection to bus bar

F (OLTC) step-up transformer (high rating)

(b)

Figure 3. Overview of system architecture with thinkable, fully optional modules (none mandatory, all to be implemented at will and at any time). (a) Exemplary specific configuration of a unit portfolio with different technologies being modularly integrated; (b) Floor layout indicating one practical implementation of the system architecture (not to scale).

On the right hand side, we find the system block for the electric connection. As for conventional generation in huge central power stations, it is likely that a generator step-up transformer (GSU) behind the busbar interfaces the station and the electric grid (Figure 3b).

In this conceptual systems perspective, it is assumed that all available conversion units are connected to both networks, i.e., electricity and district heating. Some units might additionally be connected to another supply-like gas mains, but the explanations here focus the supply perspective and thus omit such energy carriers for easier comprehension.

Therefore, once the system has been set up it is able to provide heat $\left[\dot{Q}_{t h}\right]$ to the district heating system, and to provide electric power $\left[P_{e l}, Q_{e l}\right]$ to the electric grid. While heat cannot be consumed from the DHS $\left(\dot{Q}_{t h} \stackrel{!}{\geq} 0\right)$, it is possible to convert electric power to heat by using corresponding conversion units. In this case, $P_{e l} \leq 0 \wedge Q_{e l} \leq 0$ is possible as well. For instance, electric heat pumps and immersion heaters consume electric power to provide heat. 


\subsection{Physical Modularity and Standardization of Conversion and Storage Units}

Even today, small- and medium-scale CHP units are situated in intermodal containers. However, in fact, there are many more energy-related systems which are encapsulated in intermodal containers, e.g., battery energy storage systems, thermal storage, and even static synchronous compensators (STATCOM). These containers are known as 20 or 40 feet freight containers [28]. The advantage of such a packaging is given by the easier transportation to the final destination by trucks, and by the standard equipment for handling them by cranes. This decreases costs for both decision-making and handling of novel conversion units [29].

If each unit is packed into a container, then one (conversion or storage) unit equals one module. In contrast to current practice, it is, therefore, mandatorily required by this architecture that all units are actually encapsulated in intermodal containers with identical hydraulic and electric connectors. As a consequence, installed capacity and quality of supply can easily be controlled by installing additional modules. Besides, it is also possible to replace or decommission them at any time because handling and transportation is so easy. Modularity therefore also translates into scalability, which further facilitates planning and integration.

\subsection{Integration into the Electric Grid}

As the actual mix of conversion units is not specified but depends on the specific economic and environmental conditions, the entirety of conversion units can act as a load, as an in-feed or as a neutral element at the interconnection point with the electric grid. As the successive fine-tuning of the generation portfolio should not restrict the modes of operation, a sufficient electric connection is necessary. As this paper is meant to propose a certain architectural thinking, exact numbers are not named here. Instead, it is suggested to keep future changes of requirements in mind when setting up the platform for the first time, so in general, the connection (right hand side in Figure 3) should bear a certain reserve in capacity for the most likely (i.e., already anticipated) future development to be called sufficiently large.

First of all, congestions by thermal and voltage limits of the lines should be circumvented by connecting to a sensibly high (available) voltage level. Using an independent feeder might also help mitigate line overload and interaction with other loads. Depending on regulation, it might be required from the DSO or TSO to equip the generator step-up transformer (GSU) with on-load tap changer (OLTC) capabilities to react to voltage deviations or reactive power requirements at the interface between station and electric grid.

Again, these considerations depend on the specific case. By committing to the above principles, the inherent flexibility of the conversion units to provide active and reactive power to higher voltage levels can potentially be used in the future. This can come in hand as big (inert) generators are successively disconnected from the grid, and ancillary services have to be provided by a mix of different controllable supply technologies including intermittent generation from renewable energy sources.

\subsection{Hydraulic Integration into the District Heating System}

The freedom of controlling the mode of operation in the future is also necessary for the supply of heat: A reconfigurable hydraulic setup box is used to allow serial, parallel and mixed configurations depending on the current necessities (Figure 4). The hydraulic setup is deliberately treated as a black box model. Its purpose is to ascertain the compatibility of different conversion and storage units by providing an interface to the DHS that works independent of qualities, such as temperatures and mass flow rates. The necessity is especially given if there are different kinds of conversion units [30].

The ability to reconfigure the units' thermal input and output ports is an important feature for future replacements of individual units. However, the feature is also beneficial to cope with seasonal changes of the supply temperature (which is driven by ambient temperature), and by changes in the (spatial) load distribution. Hence, such reconfigurations are likely to happen multiple times per year. 
Technically, these requirements can be fulfilled by a system of controllable valves and/or low loss headers. Both elements are readily available for different ratings and other design parameters.
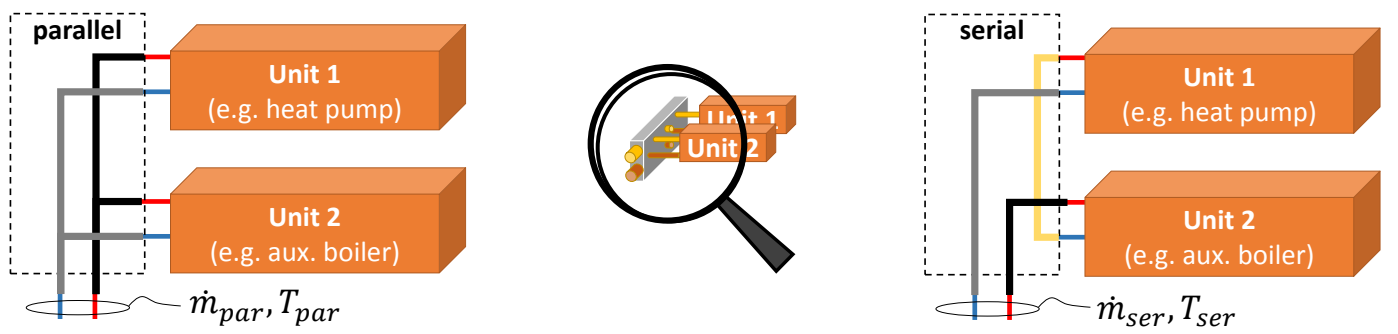

Figure 4. Example for flexible configuration of the hydraulics.

\subsection{Spatial Integration into the Urban Built Environment (Spatial Planning Perspective)}

The floor plan in Figure $3 b$ is a rather large example of existing heat station facilities. However, many cities do in fact comprise brownfields with good connectivity to electric grid and DHS. As they might be found in different locations, and in differing qualities and sizes, the general applicability for arbitrary available brownfields is visually indicated by Figure 5. It shows three floor plans which differ significantly in size while still maintaining the most important features of this architecture. The hereby proved scalability of the entire platform also indicates the possibility to split the system if necessary. For instance, if multiple units are needed to cover the local heat demand, but there is no facility available that offers enough contiguous space, then a higher number of (semi-) distributed facilities can be set up instead of a central one.

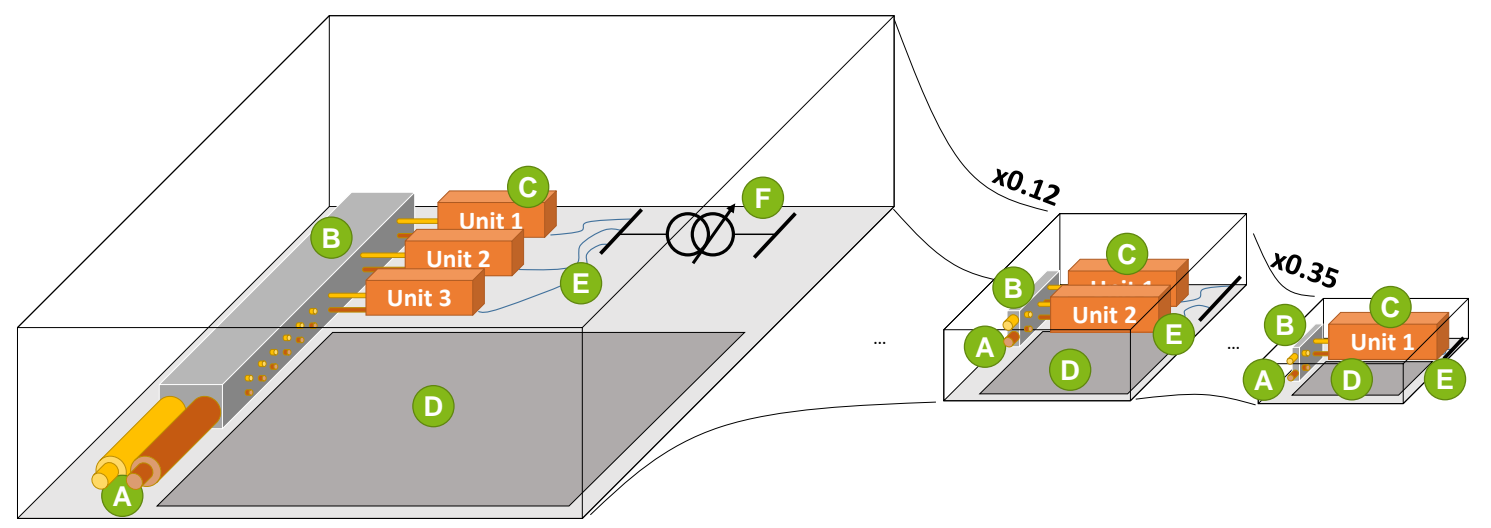

Figure 5. Three examplary configurations as a response to different spatial requirements indicate general scalability and universality of the system architecture.

To sum it up, this architecture requires to stick to the maxim of adaptability. Consequently, easy reconfiguration of the hydraulics must be possible, units must always come in intermodal containers, and extra space should be allocated for future extensions or redesign.

\section{Exemplary Hypothetical Evolution of a Realized System Over Decades}

It is assumed that a given turbine-based energy supply lacks cost-effectiveness and is thus scheduled for decommissioning. Figure 6 shows a corresponding large-scale CHP unit as it is typically found in many DHS. As soon as the facilities have been reworked according to the suggested architecture (Figure $3 b$ ), the former heat and power station has the function of an infrastructure platform. From now on, (future) supply systems can be implemented at will. 
In Figure 7, the example is broken down to five devised stages. It is furthermore assumed for this example that each of the assumed stages lasts for six to ten years, so an evolution over, e.g., 30 years is shown.

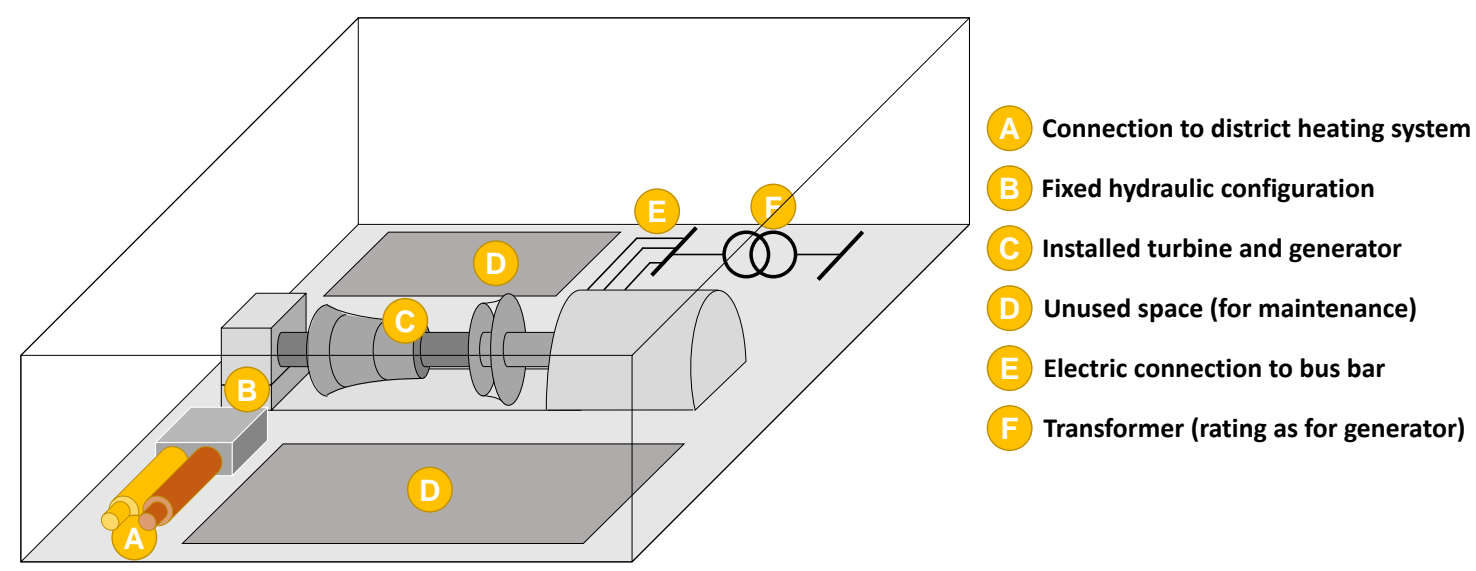

Figure 6. Typical combined heat and power generation based on a steam or gas turbine and a generator.

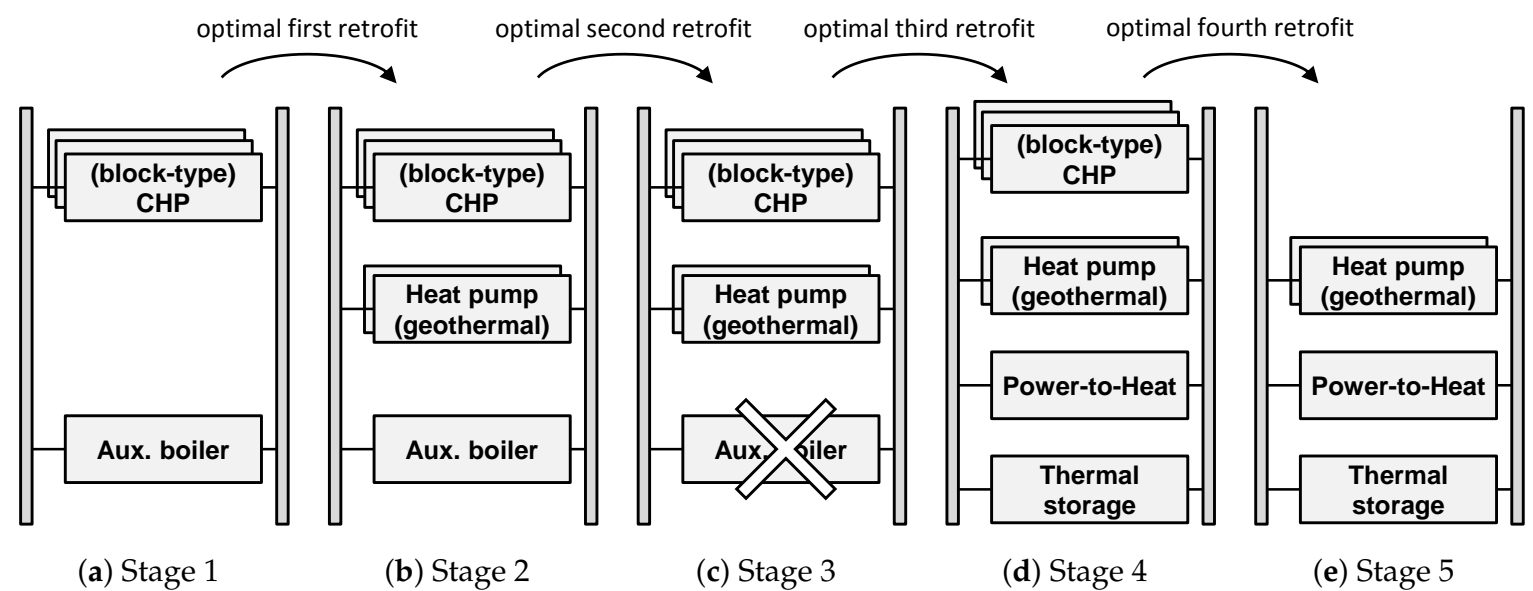

Figure 7. Assumed evolution of a configuration over years and decades.

The first transition of the system is a typical starting point (Figure 7a) that is highly realistic for today's urban energy supply. By building on three block-type CHP units and an auxiliary boiler, the implemented system is fully compatible with the requirements of the architecture, and the units are in fact cost effective in today's price and regulation regime.

In a second stage (Figure $7 \mathrm{~b}$ ), the national government has set up a fund for projects that build on geothermal heat. Therefore, in this stage, a drilling project has succeeded, and two electric heat pumps are commissioned.

In the third stage (Figure 7c), the heat demand of the stock of urban buildings has decreased significantly due to strong modernization efforts by different institutional real estate investors. Consequently, the auxiliary gas boiler (with the highest specific heat prices) is decommissioned.

The fourth stage (Figure $7 \mathrm{~d}$ ) builds on the assumption that numerous big generators are now missing in the electrical grid. Therefore, the capacity prices for ancillary services have been increased dramatically, and an investment in power-to-heat and thermal storage is finally favourable. Costs are covered by a market driven operation that takes into account the revenues from operating reserve while covering the heat demand.

In the final stage (Figure 7e), it is furthermore assumed that two effects once again change the optimal unit portfolio: On the one hand, the carbon taxation of fuels (i.e., natural gas) has 
been increased by a new ordinance for environmental reasons, on the other hand, the integration of generation from RES has progressed significantly over the past years. Of course, with the previous investments into additional installed capacity, the CHP units are obsolete now, and their operation is not cost effective. As a general overhaul is due, they are simply decommissioned, uninstalled, and not expected to be replaced.

This sample story is able to once again highlight necessity and change of optimality (as opposing to greenfield optimality assumed in [27], and discussed above), and it shows how the adaptivity of the system architecture is enabling efficient reconfigurations whenever needed.

Of course, initial retrofitting of existing facilities, or erection of the platform respectively involves (additional) up-front costs. To argue the necessity of implementing the proposed architecture, different advantages of an implementation are argued from a highly conceptual and thus more abstract perspective in the following section.

\section{Technical, Strategical and Societal Prospects of the Introduced Adaptability}

In Section 4.1, the meaning and foundation of adaptability are discussed in the context of energy supply. Based on this concept, the added value brought by the infrastructure is argued in Sections 4.2-4.5. Afterwards, thinkable actors that might benefit from such an investment are presented (Section 4.6).

\subsection{Adaptability as an Indicator for the Sustainability of Systems}

The value proposition of this architecture is best described by the word adaptability. Therefore, the following Definition 1 in line with [31] shall be used:

Definition 1 (Adaptability). Adaptability is the quality of being able to adjust to new conditions.

In the context of energy systems, the notion of adaptability involves the adjustability of (1) the (original) configuration of conversion units; (2) the specific operation; (3) the use/value that is brought by the operation of the system.

In fact, transformability was identified as a key element in factory planning [32]. The authors also identify certain enabling elements that have to be followed to ascertain transformability (in analogy to the suggested adaptability). These are called changeability enablers and comprise universality, scalability, modularity, mobility, and compatibility. The analogy to a factory as an infrastructure can thus be used to additionally discuss the architecture from factory planning perspective.

Following Table 1, it becomes clear that all aspects of transformability suggested in [32] have been implemented by the platform. However, it also becomes clear that key concepts from factory planning cannot be copied and applied one by one. Instead, a mapping is necessary, e.g., standardization is best argued by compatibility and universality, and the physical implementation even comprises three different aspects. Interestingly, the aspect of mobility is covered indirectly because of the mandatory use of intermodal containers although it is not even a key element of this architecture.

Building on this adaptability, four categories of added value can be identified, which are used to structure the following discussion:

1. Lower cost of redevelopment and redesign,

2. lower cost of installation and system integration,

3. compatibility with future markets, and

4. local concentration, economies of scale and continuous controllability. 
Table 1. Analogy to factory transformability features.

\begin{tabular}{lll}
\hline $\begin{array}{l}\text { Key Element of } \\
\text { This Architecture }\end{array}$ & Note on Physical Implementation & $\begin{array}{l}\text { Changeability } \\
\text { Enablers [32] }\end{array}$ \\
\hline $\begin{array}{l}\text { Scalability, } \\
\text { Modularity }\end{array}$ & $\begin{array}{l}\text { Block-type units in intermodal containers } \\
\text { (as defined in ISO 668 [28]) }\end{array}$ & $\begin{array}{l}\text { Scalability } \\
\text { Modularity } \\
\text { Mobility }\end{array}$ \\
\cline { 2 - 3 } & $\begin{array}{l}\text { Hydraulic matrix setup and electric grid } \\
\text { connection }\end{array}$ & Compatibility \\
\cline { 2 - 3 } & Defined connectors and outlets for all units & Inherent feature of electric power and heat \\
& (commodities) & Universality \\
\cline { 2 - 3 }
\end{tabular}

It should be noted that these categories are not mutually exclusive. Instead, they support one another and are interdependent. Figure 8 depicts the hierarchy of enabling elements of the architecture and prospects for a system operator on a high level of abstraction.

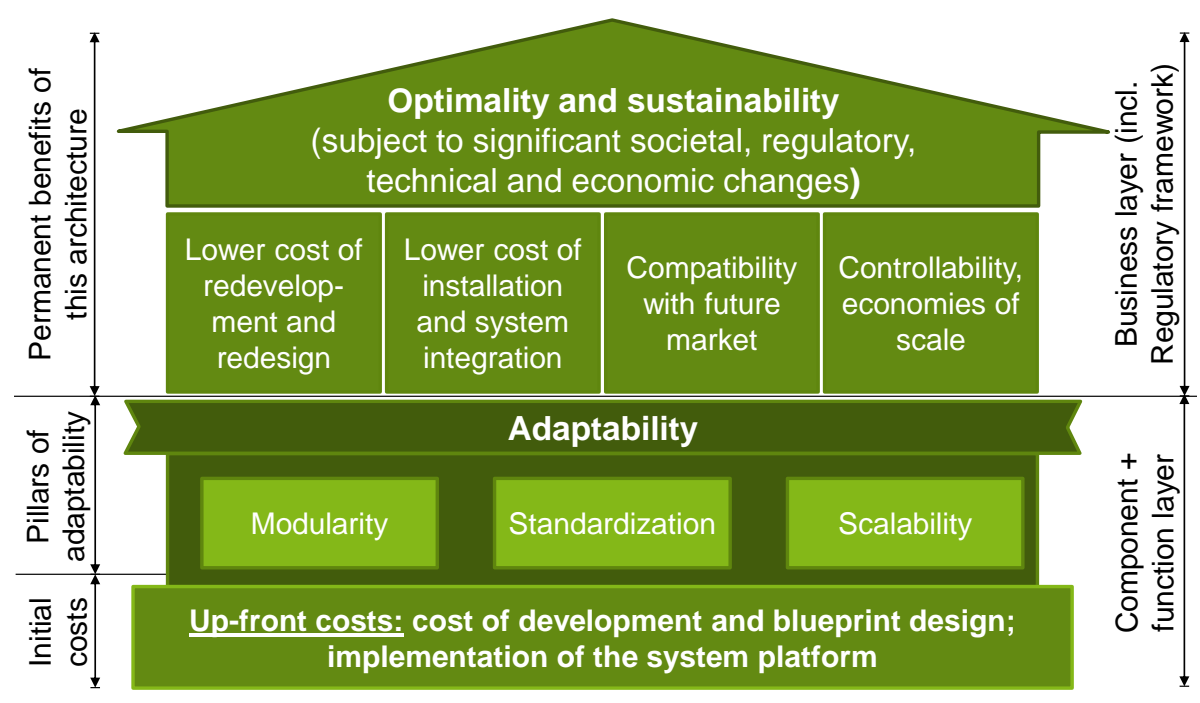

Figure 8. Conceptual view on the hierarchy of enabling elements of the infrastructure and derived benefits of an implementation.

\subsection{Lower Cost of Redevelopment and Redesign of Portfolios}

As soon as the proposed architecture is realized, it acts as a platform for future evolutions of conversion and generation. Changes to the portfolio can then be done at relatively low cost. However, each stage of redesign or optimization still has to be planned and calculated. So, the costs for planning and designing future (successive) systems rather depend on the availability of software tools and engineering knowledge in the field of energy systems.

Compatibility to numerous fields of research and existing system descriptions is in fact given (cf. Appendix A for details). This includes multi energy systems (MES) [33], distributed multi generation (DMG) [34], the energy hub [27,35], and urban energy systems (UES) [36]. Therefore, all simulation and optimization tools and studies that deal with optimal design, placement, operation, or market integration can be reused, which is an important feature to ensure low planning costs.

For instance, to connect to the introduction, the case studies in $[10,26]$ were conducted with energy hub and MES in mind. However, both approaches remain on a high abstraction level where the installation of additional units is directly possible. So, it was not discussed in their contributions if their optimal design is actually technically feasible, or if spatial constraints hinder the optimal 
implementation. In addition, even if technical feasibility was given, it is likely that high transaction costs would have been involved for an actual realization. Consequently, the here proposed architecture builds the foundation for both case studies to be rolled out in practice, and supports their optimization approaches with practical evidence of applicability, which is a strong contribution.

\subsection{Lower Cost of Installation and System Integration}

A reference case for comparison is given by decentralized installation of the same equipment in individual buildings (e.g., hospitals, residential buildings, office buildings). Due to the diversity of conditions found in buildings, which means the siting in the given space, the procurement of additional components, and even the wiring thereof has to be tailored to the conditions and expectations of the individual customer then. According to [37], this system integration accounts to additional $39 \%$ to $76 \%$ of the module cost of block-type CHP units alone. These costs do not even include planning, expert's reports or the request for bids (in a public bidding process). So, the sum of all these expense factors renders a significant share of all projects uneconomic.

However, as soon as the proposed architecture is implemented, these costs are significantly reduced. The same is true for the installation since all modules can be transported at low costs.

\subsection{Long-Term Market Compatibility}

\subsubsection{Access to New Markets}

As procurement prices differ for energy depending on the sales volume of the customer, bulk buyers have a competitive advantage in contrast to, e.g., domestic customers. According to [38], this is one of the main reasons why distributed CHP units for smaller buildings are often not cost-effective. Consequently, they depend much more on subsidies.

With this architecture, this competitive advantage is maintained, i.e., procurement of natural gas is relatively low despite the use of the units typically found for smaller scale application. In addition, certain markets are only available with a certain market power, e.g., spot markets and over-the-counter trading for electricity are possible due to the aggregation of generation capacity.

\subsubsection{Market Compatibility by Fit: Generation Capacity and Quality}

If long-term market compatibility shall be guaranteed, an increase and decrease in generation capacity must be possible in the short- to medium-term. Due to the high stress of the ICE of many CHP units, a revision or general overhaul is required every 25,000 to 35,000 operating hours [39]. Consequently, for this exemplary technology, a transition towards a different stock of units change might be possible every five to seven years (at 5000 full load hours). In practice, for a given mix of conversion units, it is likely that even in short term one unit is due for replacement. This is a direct consequence of the modularity.

Another advantage that can be drawn from the modularity is the changability of quality of supply. For instance, regulators might introduce a legal limit on thermal losses or emissions of carbon dioxide equivalents, or even the level of noise emissions. As the combined (electro-thermal) supply task in the urban built environment changes continuously [40], the ratio of electric and thermal power output (i.e., the electric CHP coefficient $\sigma_{e l}$ ) can be manipulated by switching to a different technology.

Every single requirement discussed above might generally add another dimension of quality. So, the notion of quality is much more extensive than the examples above, and it can be argued that the desired level of quality (although abstract) can generally be expected to increase. A visual concept of the dimensions of capacity and quality can also be found in Figure 9. 


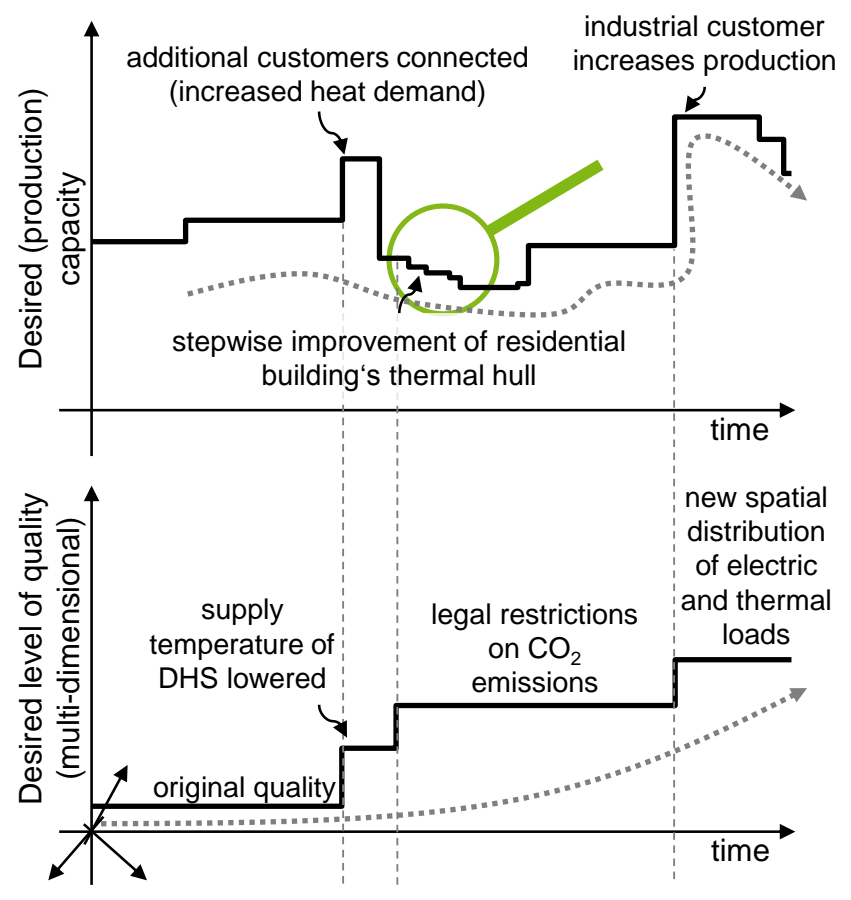

Figure 9. Qualitative difference between pure generation power and the associated quality of supply (deliberately without time and capacity scale).

\subsection{Local Concentration, Economies of Scale and Continuous Controllability}

\subsubsection{Local Concentration and Economies of Scale}

This architecture recommends to use the highest available voltage level, which can be any voltage level starting from low voltage (LV) though. However, even in this worst case scenario the bundling of conversion units to one facility leads to low costs for information and communication (ICT) and metering. If medium or high voltage grid are available for connection, all advantages of the centralized generation are maintained for this architecture. Please note that the comparison has to be drawn with a fully decentralized energy supply, which involves, for instance, rooftop PV and CHP units in the basement of buildings. Naturally, the fully decentralized scheme involves higher costs.

The improved market access discussed above can also be argued as part of the economies of scale, and different economies of scale can be identified in the fields of public bidding processes for new generation units, technical personnel (for maintenance), and in operation (and optimization thereof).

\subsubsection{Continuous Controllability by Available Real Options}

The continuous responsiveness to changes in the desired quality or capacity of supply can also be understood as a the provision of real options. There are numerous thinkable changes to the installed base of conversion units, as indicated in Figure 10. This includes (but is not limited to) additional units, fewer units, different units and a changed operation of units. It should be noted that such changes are possible at any time, although probably only conducted every five to ten years. It must also be stated that the availability of these options has an inherent value even if none of the options is ever called. The reason is that both actual losses and lost profits can be avoided by actively managing the portfolio of generation units.

As stated above, many CHP units must regularly undergo a general overhaul anyway, which means that the granularity of changes is high. This also helps to understand why there are more (and better) real options for this architecture available (Figure 10b) than for the case of conventional generation (Figure 10a). In addition, the more manageable size of changes directly leads to a 
higher financial liquidity, so both the dimension of time and corresponding financial resources can be improved.

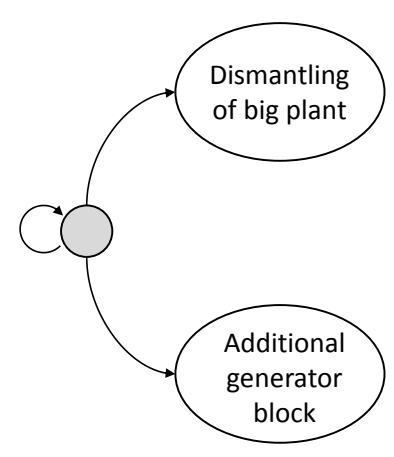

High capital intensity, long

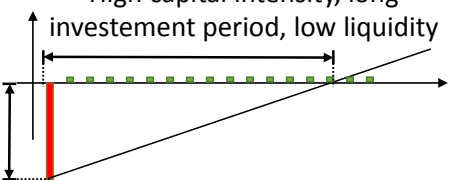

(a)

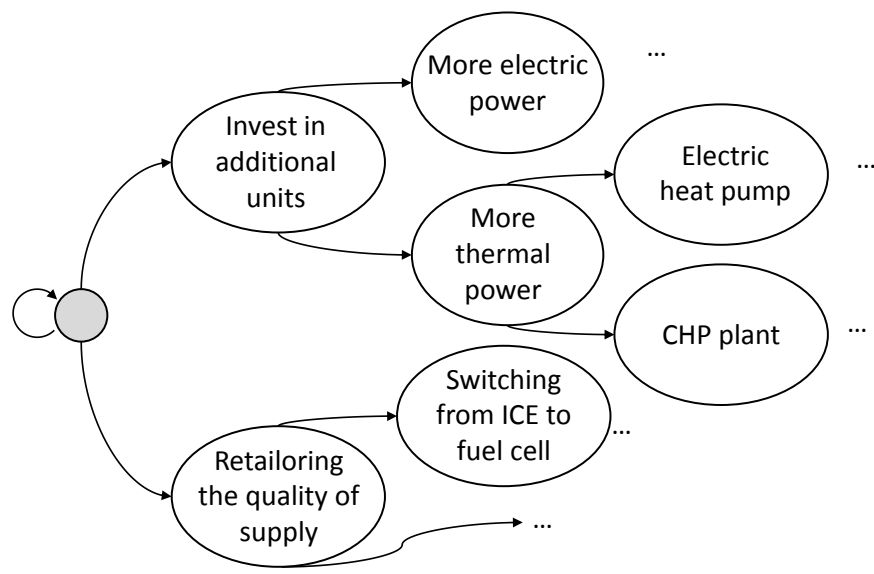

Low capital intensity, short

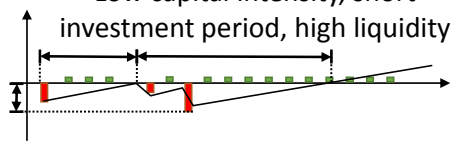

(b)

Figure 10. Availability of real options may inhibit (left) or support (right) the adaptability of a portfolio, and affects the quality of the associated investment cash flows (investment period and capital intensity). (a) Conventional generation: few real options, low liquidity; (b) This architecture: many real options, high liquidity.

The value of these real options can be roughly estimated by applying the methodology presented in [26] to a scenario of demand and economic conditions, but this is out of the scope of this paper.

\subsection{Thinkable Actors for an Implementation (and Business Cases)}

For such a platform the categories of research, funding, ownership and operation can be considered independently. Here we omit the initial research and focus on funding, ownership and operation instead:

Generating companies (GenCos) might be able to build a business case on this architecture by focusing on the by-product of heat, i.e., instead of erecting other big central generators, a certain share of the marketable electric energy might be covered locally. In comparison to the status quo, this can be thought of as cutting a portion of the current generation portfolio into manageable chunks, siting them in the urban environment (closer to heat demand), and avoiding lossy condensation in cooling towers (i.e., just feeding the DHS instead). GenCos are experts in asset management and (risk-averse) portfolio theory, so they could easily implement the system.

Municipal utilities which operate a given DHS could become trusted partners of owner-occupiers, landlords and real estate companies by offering heat and electricity which is guaranteed to be generated in an environmental-friendly way. The value proposition comes from the integration of more advanced technologies as wished-for by the customers, and the platform could serve as a local show room open to the public, so every interested customer could visit the facility to understand how the energy transition might be shaped by current and future investments into new modules.

Such a system might also be implemented as a community energy system [41] by an energy cooperative. This case is close to municipal utilities because of the interest of the customers (here: the community) to invest into a more environmental-friendly product. However, the economic feasibility might be easier to achieve due to a differing price sensitivity of involved customers: For instance, 
higher specific prices for heat and electricity might be accepted, and even a flat rate might be an option. Furthermore, cooperative shares might be drawn to collect money, and banks might grant further loans accepting these shares as a security.

In general, it is even thinkable that in the context of future energy liberalisation, such an infrastructure is deliberately opened to multiple parties, i.e., non-discriminatory access is provided to all actors mentioned above. In this scenario, the platform acts as a colocation centre (as known from data centres). This would in turn eliminate the operational challenges that go along with a provision of third party access to the DHS [42].

\section{Conclusion and Outlook}

\subsection{Summary and Conclusions}

Today's investment in new generation capacity is characterized by high capital intensity and long-term depreciation. Moreover, the risk of misinvestments and thus full write downs is significant due to a high level of uncertainty of demand, prices and regulation. Therefore, more efficient conversion technologies and systems (e.g., CHP and energy systems integration) are rarely implemented.

In this paper, the difficulty in finding a long-lasting, efficient and optimal mix of energy supply is tackled from the perspective of modular infrastructure. To this end, a paradigm change is fostered in planning and implementation is envisioned. This is achieved by a highly adaptable system architecture, which can in fact be used as a practical starting point by a wide range of stakeholders.

Necessity and added value were discussed in terms of technical, strategical and societal prospects. Sticking to a high level of abstraction (bird's eye view) presents the architecture's strengths independent of country and particular case study. Although strong focus was laid on the conceptual strength of the architecture, all advise remains specific. By sticking to the guiding principles discussed in this paper, risk-averse investors can benefit from a step-wise (more scalable and thus manageable) systems integration.

Besides, to the authors knowledge, this vision of an architecture is the first practical guide on implementation of integrated energy systems (energy hubs, DMG etc.) at the community or urban/municipality level at all, which is an important contribution.

\subsection{Outlook on Future Research}

Naturally, due to the novelty of the developed architecture, neither cost-effectiveness nor exact standardization of all connectors could be analyzed and described in detail in this paper. However, as the architecture is compatible with the energy hub, a multitude of developed optimization tools in literature can be used to analyze the depicted example case as well as comparable scenarios for municipal district heating utilities (Examples are given by $[10,26])$.

For now, it is difficult to completely assess what magnitude of adaptability should be provided today to account for future uncertainties, e.g., how much (physical) space should be left for additional units, and how high the transformer rating should be in practice. New tools thus have to be developed to find good compromises between spatial requirements, ground costs, initial investment and desirable level of adaptability (or existing tools have to be applied to this new research question). Furthermore, the standardization process for block-type conversion and storage units in intermodal containers will have to be started, which is a diligent but routine piece of work.

Despite all these necessities, the authors are confident that implementing integrated energy systems according to the developed architecture will eventually enable a faster and more cost-effective energy transition due to the enabling character of the integrated infrastructure.

Acknowledgments: This work was supported by the North-Rhine Westphalian Ministry of Culture and Science (MKW NRW) under research Grant No. 322-8.03-110-116441. We acknowledge financial support by Deutsche Forschungsgemeinschaft and Technische Universität Dortmund/TU Dortmund Technical University within the funding programme Open Access Publishing. 
Author Contributions: Jonas Hinker devised the main conceptual idea and wrote the paper. Thomas Wohlfahrt analyzed the technical feasibility of the hydraulic integration. Emily Drewing conducted a stakeholder analysis and conceived of the fit of the concept of community energy systems. Sergio Felipe Contreras Paredes investigated technical details of GSU transformers and harmonized the discussion of the concept with the terminology of microgrids where appropriate. Daniel Mayorga González improved the overall presentation of the concept for the target audience. Johanna M. A. Myrzik supervised the project. All authors discussed the presentation and commented on the manuscript.

Conflicts of Interest: The authors declare no conflict of interest.

\section{Appendix A. Analogies and Connections to Existing Architectures, System Descriptions and Concepts}

A lot of concepts were presented in literature to model energy systems integration and discuss its prospects (cf. [33] for a review). This section takes a look at the most important such architectures and conceptual frameworks to prove recent research in this field can easily be adopted, i.e., optimization and planning tools can be used further.

\section{Appendix A.1. Integrated Infrastructure and Supply Planning: Urban Energy Systems (UES)}

Urban energy systems (UES) [36] were developed as a planning tool that optimizes the location of generation facilities and their connection to loads/customers. For instance, it was applied in [43] to find the optimal siting of heat stations and CHP units in an urban (i.e., densely populated) context. Here, technical non-feasibility of certain projects was considered by additional constraints in the optimization. So, actual planning restrictions were reflected in their tool.

The architecture presented in this paper has been characterized as enabling infrastructure. It is completely in line with the aim of UES itself. In addition, as the number of options and general feasibility is increased by its implementation, it is thus clear that certain constraints can effectively be revoked.

\section{Appendix A.2. Local Multi-Carrier Generation: Distributed Multi-Generation (DMG)}

Another important contribution comes from the concept of distributed multi generation (DMG) presented in [34]. In their vision, multi generation is a widespread means implemented locally in smaller scale and in different locations. While the main goal is to cover local heat and electricity demand, certain connections are mandatory for DMG: excess heat must be injected into a DHS, a (rigid) electric grid is connected anyway, and cooling or even hydrogen network can be part of the overall system as well (if applicable). The basic idea is to require a CHP plant which can be enhanced by an additional generation plant (AGP). The AGP is not specified but shall in general complement the CHP generation. It is important to note that the hydraulic configuration is explicitly discussed, i.e., the need to engineer the optimal connection is identified.

The DMG system is thus more specific with the mandatory requirement of a CHP to be installed, but generic with regard to all other (additional) technologies. It points out the same necessities for the hydraulics that have been advanced in this architecture. Again, the additional cool and hydrogen production discussed for DMG do not contradict this work at all, but can of course be implemented as well.

\section{Appendix A.3. High Level Multi-Input-Multi-Output Systems Perspective: Energy Hub}

Another concept that has greatly contributed to the understanding of energy systems integration is the energy hub [27]. Their idea was to develop a generalized and fully linearized system description to ease the implementation for optimizations for multiple energy carriers. Furthermore, their vision was to start with a greenfield approach to find a desirable (supposed to be) optimal solution for the future. Certain transition paths should then pave the way to approach this final solution (Figure 1).

The similarity with the proposed architecture and the DMG concept is apparent, but certain differences become clear as well: The energy hub is a much more abstract multi-input-multi-output 
converter system. Even the description remains highly conceptual as the actual dimension and implementation of an energy hub is left open to a potential adopter. Instead, the general implementability for buildings, production facilities, cities or other arbitrary entities is discussed. As for DMG, one type of unit per class is considered, which is a helpful assumption for the studies of the general feasibility. However, this architecture clearly differs and advances the understanding of practical future designs of integrated energy systems by (potentially) incorporating multiple units per class. As the research of energy hubs has greatly progressed, the step towards practical implementation suggested in this paper is an important connection to current literature.

\section{References}

1. Connolly, D. Heat Roadmap Europe: Quantitative comparison between the electricity, heating, and cooling sectors for different European countries. Energy 2017, 139, 580-593.

2. Clegg, S.; Mancarella, P. Integrated Electrical and Gas Network Flexibility Assessment in Low-Carbon Multi-Energy Systems. IEEE Trans. Sustain. Energy 2016, 7, 718-731.

3. Heinen, S.; Hewicker, C.; Jenkins, N.; McCalley, J.; O'Malley, M.; Pasini, S.; Simoncini, S. Unleashing the Flexibility of Gas: Innovating Gas Systems to Meet the Electricity System's Flexibility Requirements. IEEE Power Energy Mag. 2017, 15, 16-24.

4. Liu, X.; Mancarella, P. Modelling, assessment and Sankey diagrams of integrated electricity-heat-gas networks in multi-vector district energy systems. Appl. Energy 2016, 167, 336-352.

5. O'Malley, M.; Kroposki, B. Unlocking Flexibility: Energy Systems Integration [Guest Editorial]. IEEE Power Energy Mag. 2017, 15, 10-14.

6. Good, N.; Martínez Ceseña, E.A.; Mancarella, P. Business Cases. In Energy Positive Neighborhoods and Smart Energy Districts; Elsevier: Amsterdam, The Netherlands, 2017; pp. 159-226.

7. Magnusson, D. Swedish district heating-A system in stagnation: Current and future trends in the district heating sector. Energy Policy 2012, 48, 449-459.

8. DeLlano Paz, F.; Calvo-Silvosa, A.; Antelo, S.I.; Soares, I. Energy planning and modern portfolio theory: A review. Renew. Sustain. Energy Rev. 2017, 77, 636-651.

9. Ioannou, A.; Angus, A.; Brennan, F. Risk-based methods for sustainable energy system planning: A review. Renew. Sustain. Energy Rev. 2017, 74, 602-615.

10. Favre-Perrod, P.; Kienzle, F.; Andersson, G. Modeling and design of future multi-energy generation and transmission systems. Eur. Trans. Electr. Power 2010, 20, 994-1008.

11. Carpaneto, E.; Chicco, G.; Mancarella, P.; Russo, A. A Decision Theory Approach to Cogeneration Planning in the Presence of Uncertainties. In Proceedings of the 2007 IEEE Lausanne Power Tech, Lausanne, Switzerland, 1-5 July 2007; pp. 1435-1440.

12. Thangavelu, S.R.; Khambadkone, A.M.; Karimi, I.A. Long-term optimal energy mix planning towards high energy security and low GHG emission. Appl. Energy 2015, 154, 959-969.

13. Baringo, L.; Conejo, A.J. Risk-Constrained Multi-Stage Wind Power Investment. IEEE Trans. Power Syst. 2013, 28, 401-411.

14. Bruno, S.; Ahmed, S.; Shapiro, A.; Street, A. Risk neutral and risk averse approaches to multistage renewable investment planning under uncertainty. Eur. J. Oper. Res. 2016, 250, 979-989.

15. Mashayekh, S.; Stadler, M.; Cardoso, G.; Heleno, M. A mixed integer linear programming approach for optimal DER portfolio, sizing, and placement in multi-energy microgrids. Appl. Energy 2017, 187, 154-168.

16. Wierzbowski, M.; Lyzwa, W.; Musial, I. MILP model for long-term energy mix planning with consideration of power system reserves. Appl. Energy 2016, 169, 93-111.

17. Dominguez, R.; Conejo, A.J.; Carrion, M. Investing in Generation Capacity: A Multi-Stage Linear-Decision-Rule Approach. IEEE Trans. Power Syst. 2016, 31, 4784-4794.

18. Moshi, G.G.; Bovo, C.; Berizzi, A.; Taccari, L. Optimization of integrated design and operation of microgrids under uncertainty. In Proceedings of the 2016 Power Systems Computation Conference (PSCC), Genoa, Italy, 20-24 June 2016; pp. 1-7.

19. Von Haebler, J.; Erlemeyer, F.; Rehtanz, C.; Blanco, G. Modelling of efficient distributed generation porfolios using a multiobjective optimization approach. In Proceedings of the 2017 IEEE Manchester PowerTech, Manchester, UK, 18-22 June 2017; pp. 1-6. 
20. Wang, H.; Huang, J. Cooperative Planning of Renewable Generations for Interconnected Microgrids. IEEE Trans. Smart Grid 2016, 7, 2486-2496.

21. Chis, A.; Koivunen, V. Coalitional game based cost optimization of energy portfolio in smart grid communities. IEEE Trans. Smart Grid 2017, 1.

22. Franco, J.F.; Rider, M.J.; Romero, R. Robust Multi-Stage Substation Expansion Planning Considering Stochastic Demand. IEEE Trans. Power Syst. 2016, 31, 2125-2134.

23. Huang, Y.H.; Wu, J.H.; Hsu, Y.J. Two-stage stochastic programming model for the regional-scale electricity planning under demand uncertainty. Energy 2016, 116, 1145-1157.

24. Boomsma, T.K.; Meade, N.; Fleten, S.E. Renewable energy investments under different support schemes: A real options approach. Eur. J. Oper. Res. 2012, 220, 225-237.

25. Min, K.J.; Wang, C.H. Generation planning for inter-related generation units: A real options approach. In Proceedings of the 2000 Power Engineering Society Summer Meeting (Cat. No.00CH37134), Seattle, WA, USA, 16-20 July 2000; pp. 2261-2265.

26. Martínez Ceseña, E.A.; Capuder, T.; Mancarella, P. Flexible Distributed Multienergy Generation System Expansion Planning Under Uncertainty. IEEE Trans. Smart Grid 2016, 7, 348-357.

27. Geidl, M.; Koeppel, G.; Favre-Perrod, P.; Klockl, B.; Andersson, G.; Frohlich, K. Energy hubs for the future. IEEE Power Energy Mag. 2007, 5, 24-30.

28. International Organization for Standardization. ISO 668:2013 Series 1 Freight Containers—Classification, Dimensions and Ratings; International Organization for Standardization: Geneva, Switzerland, 2013.

29. Martin, C. The Packaging of Efficiency in the Development of the Intermodal Shipping Container. Mobilities 2014, 9, 432-451.

30. Van Erdeweghe, S.; van Bael, J.; Laenen, B.; D'haeseleer, W. "Preheat-parallel" configuration for low-temperature geothermally-fed CHP plants. Energy Convers. Manag. 2017, 142, 117-126.

31. Stevenson, A. Oxford Dictionary of English, 3rd ed.; Oxford University Press: Oxford, UK, 2011.

32. Wiendahl, H.P.; ElMaraghy, H.A.; Nyhuis, P.; Zäh, M.F.; Wiendahl, H.H.; Duffie, N.; Brieke, M. Changeable Manufacturing - Classification, Design and Operation. CIRP Ann. 2007, 56, 783-809.

33. Mancarella, P. MES (multi-energy systems): An overview of concepts and evaluation models. Energy 2014, 65, 1-17.

34. Chicco, G.; Mancarella, P. Distributed multi-generation: A comprehensive view. Renew. Sustain. Energy Rev. 2009, 13, 535-551.

35. Geidl, M. Integrated Modeling and Optimization of Multi-Carrier Energy Systems. Ph.D. Thesis, ETH Zürich, Zürich, Switzerland, 2007.

36. Keirstead, J.; Shah, N. Urban Energy Systems: An Integrated Approach; Routledge: Abingdon, UK, 2013.

37. ASUE. BHKW-Kenndaten 2014/2015: Module, Anbieter, Kosten; ASUE: Yerevan, Armenia, 2014.

38. Evans, R. Environmental and economic implications of small-scale CHP. Energy Policy 1993, 21, 79-91.

39. Lantz, M. The economic performance of combined heat and power from biogas produced from manure in Sweden-A comparison of different CHP technologies. Appl. Energy 2012, 98, 502-511.

40. Hinker, J.; Myrzik, J.M.A.; Witte, N.; Heinzel, A. Analysis of the interdependencies of the socio-technical parameters of energy supply in urban quarters. In Proceedings of the Internet der Dinge-VDE Kongress 2016, Mannheim, Germany, 7-8 November 2016.

41. Hoffman, S.M.; High-Pippert, A. From private lives to collective action: Recruitment and participation incentives for a community energy program. Energy Policy 2010, 38, 7567-7574.

42. Söderholm, P.; Wårell, L. Market opening and third party access in district heating networks. Energy Policy 2011, 39, 742-752.

43. Keirstead, J.; Samsatli, N.; Shah, N.; Weber, C. The impact of CHP (combined heat and power) planning restrictions on the efficiency of urban energy systems. Energy 2012, 41, 93-103.

(C) 2018 by the authors. Licensee MDPI, Basel, Switzerland. This article is an open access article distributed under the terms and conditions of the Creative Commons Attribution (CC BY) license (http:/ / creativecommons.org/licenses/by/4.0/). 